\title{
Confocal Blue Reflectance Imaging in Type 2 Idiopathic Macular Telangiectasia
}

\author{
Peter Charbel Issa, ${ }^{1}$ Tos T. J. M. Berendschot, ${ }^{2}$ Giovanni Staurenghi, ${ }^{3}$ Frank G. Holz, ${ }^{1}$ and \\ Hendrik P. N. Scholl ${ }^{1}$
}

Purpose. To report the characteristics of confocal blue reflectance imaging in type 2 idiopathic macular telangiectasia (type 2 IMT).

Methods. In a prospective observational cross-sectional study, both eyes of 33 patients with type 2 IMT were examined by means of fundus biomicroscopy, fundus photography, fluorescein angiography, and optical coherence tomography (OCT). Confocal blue reflectance (CBR) imaging was performed using a confocal scanning laser ophthalmoscope (HRA2; Heidelberg Engineering, Heidelberg, Germany). To compare the results derived from different imaging modalities, an analysis was performed using image analysis software (Heidelberg Eye Explorer; Heidelberg Engineering).

Results. CBR imaging revealed a parafoveal area of increased reflectance that was slightly larger than the area of hyperfluorescence in late-phase fluorescein angiography. The area usually encompassed an oval parafoveal area, but sectors could be spared. A parafoveal area of increased CBR was detected in $98 \%$ of eyes that showed angiographic evidence for type 2 IMT.

Conclusions. CBR imaging is a new, noninvasive, and sensitive method that may contribute to differentiate type 2 IMT from other diseases. Abnormalities of macular pigment distribution and Müller cell pathology may contribute to the phenomenon of increased CBR and thus the pathophysiology of type 2 IMT. (Invest Ophthalmol Vis Sci. 2008;49:1172-1177) DOI: 10.1167/iovs.07-0636

$\mathrm{T}$ ype 2 idiopathic macular telangiectasia (type 2 IMT) is a rare condition that usually manifests with a slow decrease in visual acuity, metamorphopsia, or both in the fifth to seventh decade. ${ }^{1-4}$ In larger cohorts, mean visual acuity has been reported to be $20 / 40,2,5$ but it may decline to $20 / 200$ and less because of secondary vascular membranes or foveal atrophy. Intraretinal pigment clumping was suggested to be a late sign

From the ${ }^{1}$ Department of Ophthalmology, University of Bonn, Bonn, Germany; the ${ }^{2}$ University Eye Clinic Maastricht, Mastricht, The Netherlands; and the ${ }^{3}$ Eye Clinic Department of Clinical Science "Luigi Sacco," University of Milan, Milan, Italy.

Presented in part at the annual meeting of the Association for Research in Vision and Ophthalmology, Fort Lauderdale, Florida, May 2007.

Supported by the Lowy Medical Research Institute (The Macular Telangiectasia Project, www.mactelresearch.com), DFG Heisenberg Fellowship SCHO 734/2-1, EU FP6, and Integrated Project "EVI-GENORET" (LSHG-CT-2005-512036).

Submitted for publication May 30, 2007; revised September 25, 2007; accepted January 16, 2008.

Disclosure: P. Charbel Issa, None; T.T.J.M. Berendschot, None; G. Staurenghi, None; F.G. Holz, None; H.P.N. Scholl, None

The publication costs of this article were defrayed in part by page charge payment. This article must therefore be marked "advertisement" in accordance with 18 U.S.C. $\$ 1734$ solely to indicate this fact.

Corresponding author: Hendrik P. N. Scholl, Department of Ophthalmology, University of Bonn, Ernst-Abbe-Strasse 2, D-53127 Bonn, Germany; hendrik.scholl@ukb.uni-bonn.de. of the disease preceding the development of neovascular membranes. ${ }^{2}$ Parafoveal scotomas were recently described as characteristic functional defects in type 2 IMT, especially in late stages of the disease. ${ }^{5}$

Fluorescein angiography has been the diagnostic gold standard in type 2 IMT, showing a diffuse hyperfluorescence in the late phase predominantly temporal to the fovea. Parafoveal telangiectatic capillaries may be seen in the early angiographic phase. Without fluorescein angiography, diagnosis of type 2 IMT can be challenging, and many patients possibly remain undiagnosed.

In the past, there have been no treatment options with proven benefit for patients with type 2 IMT. However, recent reports could show a positive effect after intravitreal application of triamcinolone ${ }^{6-8}$ or bevacizumab (an antagonist to vascular endothelial growth factor [VEGF]), ${ }^{9-13}$ and after posterior juxtascleral administration of anecortave acetate. ${ }^{14}$ With emerging new treatment options, fast, safe, and uninvasive imaging techniques for diagnosis and follow-up are needed. In this regard, a first step was to show that optical coherence tomography (OCT) shows specific changes in type 2 IMT and might therefore have a diagnostic value. ${ }^{1,15-18}$

In a recent brief report, an increased confocal blue reflectance (CBR) at $488 \mathrm{~nm}$ was shown in two patients with type 2 IMT. ${ }^{19}$ Decreased absorption or increased reflection of the blue light was suggested to cause the phenomenon. We further pursued this uninvasive diagnostic approach and suggest it to be a sensitive tool to identify patients with this rare disease.

\section{Patients and Methods}

Thirty-three patients with type 2 IMT underwent complete ophthalmic examination, including slit lamp examination with indirect ophthalmoscopy, fluorescein angiography by confocal scanning laser ophthalmoscopy (cSLO; HRA2; Heidelberg Engineering, Heidelberg, Germany), fundus photography (FF450; Zeiss, Jena, Germany), and OCT imaging (Stratus OCT; Carl Zeiss Meditec, Jena, Germany).

CBR images at a wavelength of $488 \mathrm{~nm}$ (argon laser) were obtained using the HRA2. The confocal pinhole of our system had a diameter of $100 \mu \mathrm{m}$ and a depth of focus (full width at half maximum) of 4.1 diopters (D), resulting in a drop of the detected reflectance signal of about twofold at approximately 2-D defocus. The appearance of a normal fundus in CBR imaging is shown in Figure 1A. In eight patients, additional stereo images were obtained by rotating the camera without making any vertical adjustment or movement of the fixation device. Stereo images were viewed using a stereoscopic viewer (Stereo-Vu; PS Manufacturing, Portland, OR) that allows fusion of the two images into one stereoscopic image.

Macular pigment optical density (MPOD) was assessed using a modified confocal HRA classic (Heidelberg Engineering), as described. ${ }^{20,21}$ Briefly, the instrument uses two argon excitation wavelengths at $\Lambda_{1}=488 \mathrm{~nm}$ and $\Lambda_{2}=514 \mathrm{~nm}$, in conjunction with a barrier filter that blocks all wavelengths shorter than $560 \mathrm{~nm}$. The system software generates an averaged autofluorescence image from two movies (each consisting of 16 images) acquired with each of the two excitation wavelengths. Macular pigment absorbs light in the short 
Figure 1. (A) Confocal blue reflectance image acquired with the confocal scanning laser ophthalmoscope HRA2 in a healthy subject. (B) Normal macular pigment distribution in a healthy subject (acquired with a modified HRA classic).
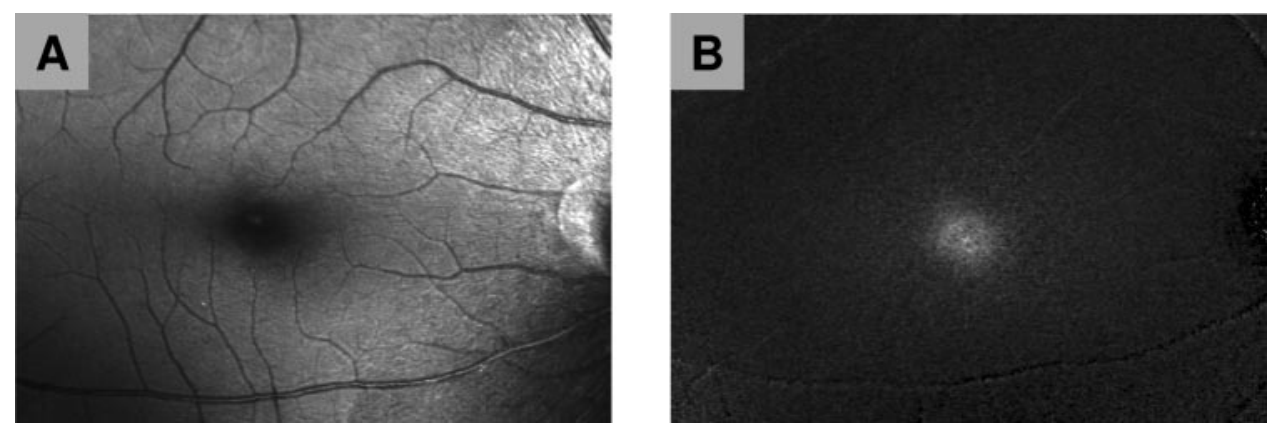

wavelength range, with a maximum at $460 \mathrm{~nm}$. Therefore, the autofluorescence image at $488 \mathrm{~nm}$ shows a strong dependence on macular pigment density because the excitation wavelength at $488 \mathrm{~nm}$ is absorbed considerably more than at $514 \mathrm{~nm}$. This results in reduced autofluorescence at $488 \mathrm{~nm}$ with increasing macular pigment density. Subtraction of the two averaged images results in an image representing the MPOD. A normal macular pigment distribution is shown in Figure 1B.

Images were analyzed using image analysis software (Heidelberg Eye Explorer [HEE]; Heidelberg Engineering). Using anatomic landmarks such as retinal blood vessels, the software allows outlining a region of interest manually and overlying the outline with a second image of the same eye. The region of interest is defined by a white line in the respective figures, enabling comparison of the size of the area of interest.

The study adhered to the tenets of the Declaration of Helsinki, and it was approved by the local ethics committee of the University of Bonn. Informed consent was obtained from all patients.

\section{Results}

The mean age of the patients was 63.3 years (SD, 6.7 years; range, 49-74 years). Seventeen patients were women, and 16 were men. Of the 66 eyes under consideration, four eyes were excluded from analysis because poor image quality (Fig. 2). There were 40 eyes without and 18 with intraretinal pigment clumping. Three eyes had a secondary vascular membrane, and one eye had undergone vitreoretinal surgery for a full-thickness macular hole (Fig. 2).

In 58 of the 62 eyes (94\%), there was an apparent increased reflectance in CBR imaging within the parafoveal area. When viewing the stereo images with a stereoscopic viewer, the reflectance appeared to be located at the level of the outer retina. The strong reflectance in the parafovea was confined by a ringlike area with a lower reflectance. Eccentric to this ring, there was a normal reflectance that in most cases appeared as strong as in the parafovea (Figs. 3-5).

Increased parafoveal reflectance in CBR imaging was related to the area of reduced retinal transparency (if visible on fundus photography; Fig. 3) and to the area of diffuse hyperfluorescence in late-phase fluorescein angiography (Figs. 3, 4). However, increased parafoveal CBR was also present in parafoveal areas with normal retinal transparency. In one eye, CBR and fundus color images were available before and after treatment with intravitreal bevacizumab. There was a normalization of the parafoveal neurosensory transparency and a marked regression of the parafoveal leakage after treatment. However, the increased parafoveal CBR was persistent in the follow-up examination (data not shown).

The area of increased CBR was usually larger than the area of angiographic leakage (Figs. 3, 4). Angiographic parafoveal hyperfluorescence at 10 minutes after dye injection was confined to the temporal two quadrants in 21 of the 55 nonproliferative eyes (38\%) with increased CBR. However, parafoveal increased CBR was confined to the temporal two quadrants in only four of those 55 eyes $(7 \%)$ but extended through three or four quadrants in the remaining. Therefore, increased CBR overlapping the vertical meridian (through the fovea) could often be observed even if the angiographic hyperfluorescence was limited to a sector temporal to the fovea (Fig. 4B). The area of late-phase fluorescein angiographic hyperfluorescence could approximate the area of increased CBR (Fig. 4D) but never exceeded it.
FIGURE 2. Diagram showing the number of eyes with type 2 IMT with and without increased parafoveal confocal blue reflectance, respectively. The bottom boxes further characterize the eyes according to clinical findings. FTMH, full thickness macular hole.

\section{All examined eyes}

$\mathrm{n}=66$

$\downarrow-n=4$ (eyes excluded due to poor image quality)

$\mathrm{n}=62$

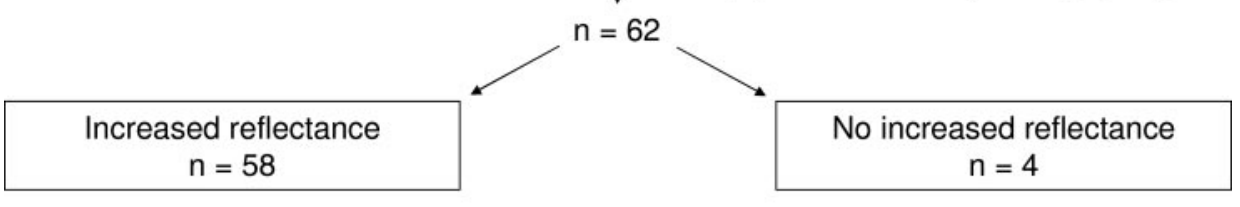

- 38 eyes without intraretinal pigment

- 17 eyes with intraretinal pigment

- 3 eyes with a neovascular membrane
- 2 eyes without intraretinal pigment and without angiographic leakage

- 1 eye with intraretinal pigment

- 1 eye with a FTMH 


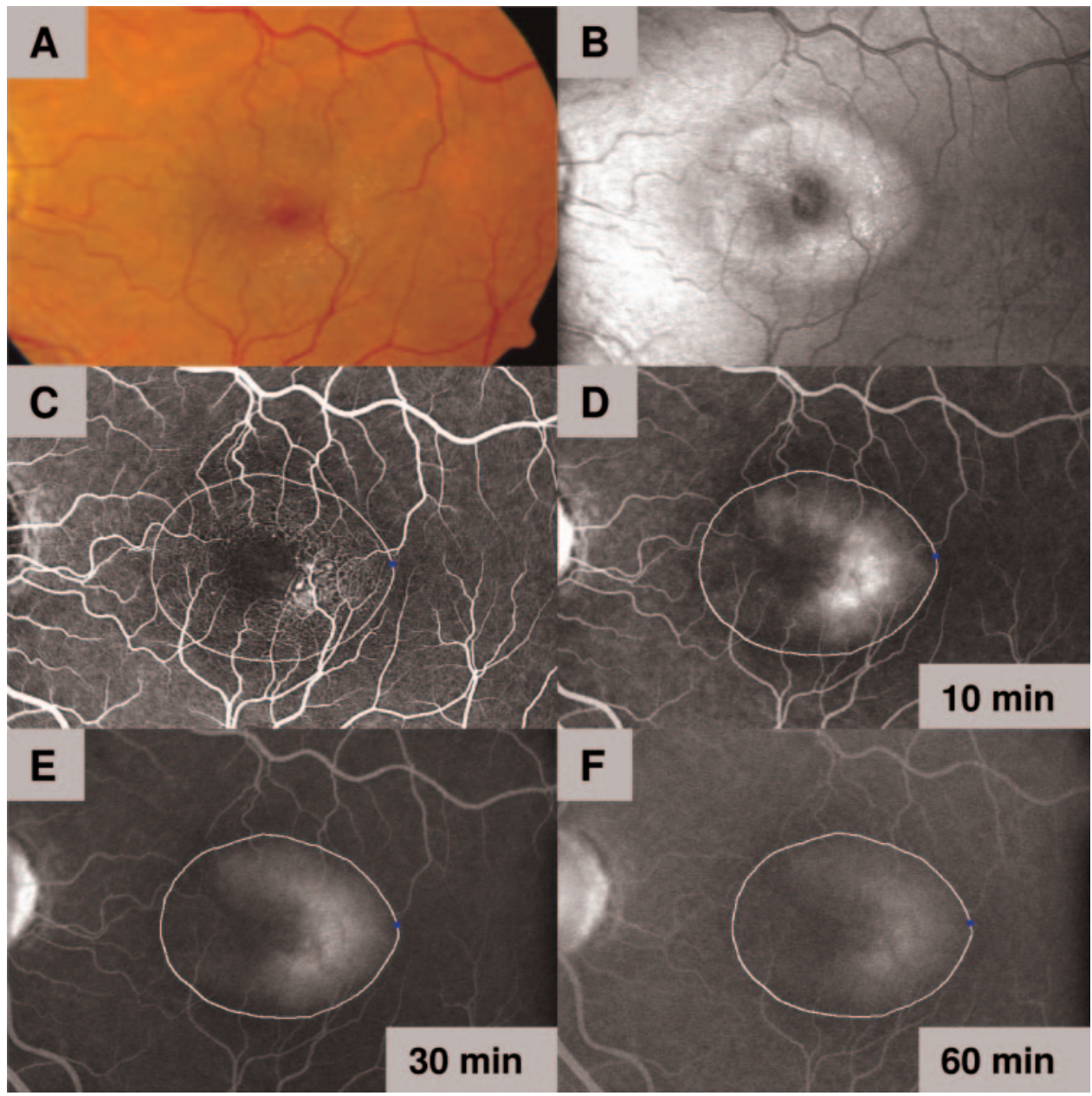

Figure 3. Left eye of a 59-year-old female patient with type 2 IMT. (A) Fundus color photography shows parafoveal loss of retinal transparency and superficial crystalline retinal deposits predominantly temporal inferior to the fovea. (B) CBR image shows strong parafoveal reflectance and crystalline deposits. The fovea appears darker. (C, D) Early- and latephase (10-minute) fluorescein angiography confirms the diagnosis of type 2 IMT. (E, F) Late phases at 30 and 60 minutes after fluorescein injection show only a minimal increase of the hyperfluorescence compared with the image at 10 minutes. Note the larger area of increased CBR, which is defined by the white line overlaid on the angiographic images.
In six patients, additional fluorescein angiographic images were obtained 30 and 60 minutes after intravenous injection of fluorescein. The area of late-phase diffuse hyperfluorescence showed either no increase or only a minimal increase compared with the involved area at 10 minutes (Fig. 3) and was still smaller than the area of increased CBR in all cases.

The parafoveal area of increased CBR had a horizontal oval shape, sometimes sparing sectors predominantly on the nasal side. In all eyes, the fovea appeared darker than the surrounding reflectance, sometimes with a slightly patchy appearance and a specular reflex. This darker spot was round in most cases but could also have a slightly irregular shape.

Increased CBR could be evenly distributed within the concerned parafoveal area (Figs. 4A, 4B) but could also show enhanced reflectance mainly at the outer border of the involved area (Fig. 4C). However, some eyes also exhibited an
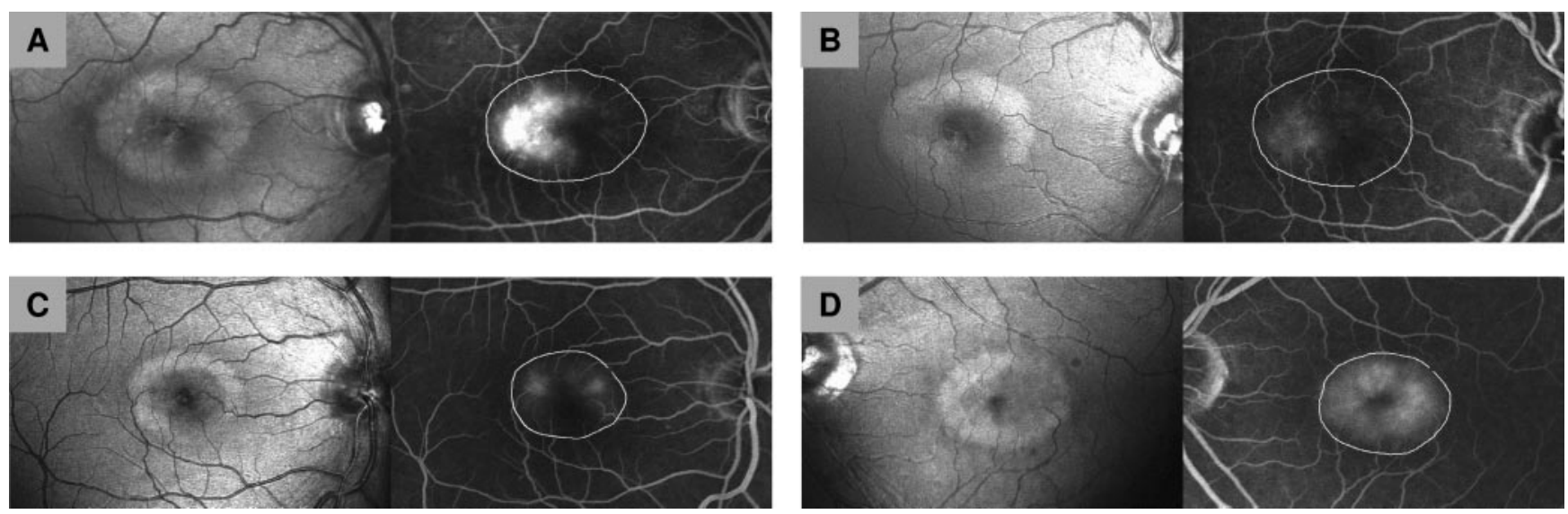

FIGURE 4. CBR images and corresponding late-phase (10-minute) fluorescein angiography in eyes with type 2 IMT. The white delineation line overlaid on the angiographic images marks the area of increased parafoveal CBR, which was always larger than the area of late-phase diffuse hyperfluorescence. Increased CBR may be evenly distributed $(\mathbf{A}, \mathbf{B})$ or ringlike (C), or it may exhibit both features (D). 


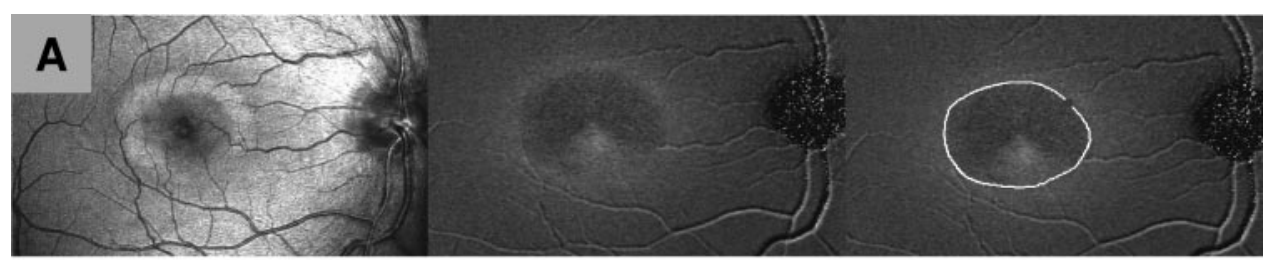

Figure 5. Comparison of the areas of increased confocal blue reflectance (CBR, left), macular pigment optical density (MPOD, middle), and MPOD image with superimposed delineation of the area of increased CBR $($ right) in four eyes of four different patients. The white delineation in the right column marks the area of increased CBR. The area of increased CBR and reduced MPOD has the same size and location. (A) The sector with preserved MPOD shows no increase in CBR. (B) The slightly preserved central MPOD results in a larger central dark spot in CBR imaging.
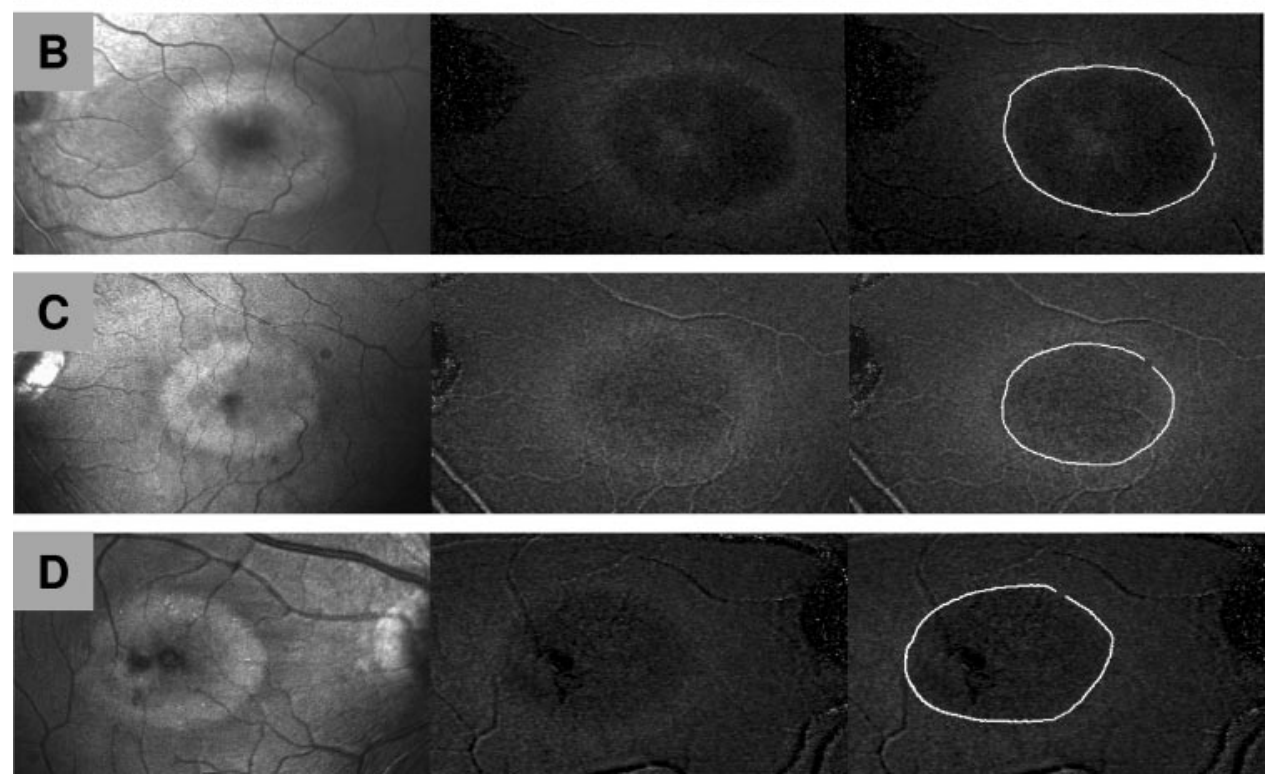

evenly distributed increased CBR in one part of the parafoveal lesion and an enhanced reflectance at the outer border in the other part of the lesion (Fig. 4D). Furthermore, a subset of eyes revealed an increased reflectance directly adjacent to the darkappearing foveal area (e.g., Figs. 4A, 4B)

In four eyes, the MPOD was compared with the CBR images acquired on the same day (Fig. 5). MPOD was reduced parafoveally, and there was a preserved MPOD ring at $5^{\circ}$ to $7^{\circ}$ eccentricity. The area of increased CBR showed the same size and shape as the area of reduced MPOD. Again, this area was larger than the area of leakage in late-phase fluorescein angiography.

Four eyes did not show increased CBR. Two of those were fellow eyes of patients with typical early-stage type 2 IMT in the other eye. These fellow eyes did also not show parafoveal hyperfluorescence in late fluorescein angiography. We hypothesize that these patients did not have monocular disease but rather had too few pathologic changes to be detected in the fellow eye. Moreover, no increased CBR was observed in the eye that had undergone vitreoretinal surgery for a full-thickness macular hole and in one eye with intraretinal pigment clumping (Fig. 2).

\section{Discussion}

This study presents a noninvasive method that may be beneficial in the differential diagnosis of type 2 IMT, confirming an initial observation. ${ }^{19}$ Increased CBR within the parafoveal area was found in all but one (the eye with intraretinal pigment clumping; Fig. 2) eyes (98\%) that showed angiographic evidence for type 2 IMT. Therefore, CBR imaging might be almost as sensitive as fluorescein angiography to detect type 2 IMT. The method might especially be helpful in early stages of disease, which are the most difficult to detect clinically. We have performed CBR imaging in patients with other macular diseases and so far have never observed the presented pattern of increased parafoveal CBR. However, the specificity of the phenomenon remains to be determined in future systematic studies.

We used an SLO to show the increased reflectance because it provides retinal images with high contrast given its confocal nature. A regular fundus camera also detects light scattered at other structures in the eye (e.g., lens, vitreous), which dilutes the signal reflected at the retina. This makes a fundus camera less suited to study the reflectance of blue light (compared with longer wavelengths) because light scattering increases with a decrease in wavelength.

As in most imaging techniques, assessment of CBR imaging depends on the experience and interpretation of the examiner. Two recent reports studying OCT findings in type 2 IMT found characteristic hyporeflective foveal spaces to be present in $56 \%$ and $80 \%$ of eyes with type 2 IMT, respectively. ${ }^{15,18} \mathrm{New}$ noninvasive imaging techniques might not substitute for angiography, but their combination may result in a high diagnostic sensitivity.

Most interestingly, the parafoveal area of increased CBR is usually larger than the area of diffuse hyperfluorescence in late-stage fluorescein angiography. This suggests a disease process that goes beyond the margins of the angiographically apparent leakage. Moreover, it would suggest that the angiographically visible alterations could be a secondary phenomenon. A retinal disease process, such as retinal dystrophy, could lead to vascular changes through the local expression of mediators (e.g., VEGF) targeting the macular capillaries.

The nature of the phenomenon of increased CBR remains to be determined. One possibility might be that the described lack of macular pigment ${ }^{4}$ results in reduced absorption and increased reflectance in the area involved. Light scattering in the blue light range was found to make only a small contribution to the absorption by the macular pigment in retinal prep- 
arations in vitro. ${ }^{22}$ Therefore, absorption and reflection mainly contribute to the appearance of the macula in blue light. The absorption maximum of macular pigment is in the range of blue light at approximately $460 \mathrm{~nm}^{22}$; therefore, reduced MPOD would result in reduced absorption of the light used in CBR imaging. The similar intensity of CBR in parafoveal areas and external to the darker ring might be explained by the decreasing density of macular pigment with increasing eccentricity. The darker-appearing rim surrounding the increased CBR could represent the ringlike area with preserved macular pigment.

The lack of macular pigment usually also encompasses the fovea, where CBR is not markedly increased. A dark spot in the central macula has been described as a normal finding in spectral reflectance of the retina with blue light, ${ }^{23}$ This may be attributed to the absence of nerve fibers at the fovea that have a maximum reflectance in the blue wavelength range. ${ }^{24} \mathrm{How}$ ever, in some cases, the dynamic range of the imaging system might not be high enough to detect reflectance changes. The slightly irregular appearance of the fovea in some images might be the result of the disturbed foveal anatomy known from OCT examinations of patients with type 2 IMT, resulting in irregular and specular reflectance patterns. If the described findings could be explained by the lack of macular pigment, CBR imaging would be an easy and widely available tool to indirectly assess a loss of macular pigment in type 2 IMT.

In the normal fundus, fine striations at the inner surface of the retina have been interpreted as nerve fiber bundles (bright striation) compartmentalized by Müller cell processes (dark striation). ${ }^{24,25}$ Franze et al. ${ }^{26}$ recently suggested that Müller cells do not reflect but rather transmit light through the retina. Interruption of this mechanism could reduce transmission and possibly increase reflectance. Therefore, pathology of Müller cells could contribute to the observed increased CBR.

A further explanation for the increased CBR could be the deposition of highly reflective material, such as crystallized macular pigment, in the neurosensory layers. Canthaxanthin retinopathy could serve as a model. Oral ingestion of canthaxanthin (a carotenoid with a molecular structure similar to that of the macular pigments lutein and zeaxanthin) for bronzing of the skin may lead to a retinopathy that shares clinical features with type 2 IMT, such as parafoveal crystalline deposits in the inner neurosensory retina ${ }^{27-29}$ (with only the largest crystals visible clinically ${ }^{30}$ ) and degenerative retinal changes. ${ }^{27} \mathrm{~A}$ presumed defect in parafoveal storage or metabolism of macular pigments in type 2 IMT could be associated with the formation of crystal-like deposits not visible on ophthalmoscopy and could therefore explain the increased reflectance observed in type 2 IMT.

Eventually, the underlying cause of the increased parafoveal CBR in type 2 IMT remains to be determined. Most evidence seems to be available for the assumption that a lack of macular pigment is responsible for the phenomenon. Other possible explanations, such as deposition of highly reflective material, should be addressed in future studies. New insights may be provided by simultaneous cSLO and high-resolution OCT imaging that allow the correlation of pathologic findings in topographic and tomographic images.

\section{Acknowledgments}

The authors thank François C. Delori for very useful discussion and critical suggestions and Marion Platen for excellent technical assistance.

\section{References}

1. Yannuzzi LA, Bardal AM, Freund KB, Chen KJ, Eandi CM, Blodi B. Idiopathic macular telangiectasia. Arch Ophthalmol. 2006;124: $450-460$

2. Gass JD, Blodi BA. Idiopathic juxtafoveolar retinal telangiectasis: update of classification and follow-up study. Ophthalmology. 1993;100:1536-1546.

3. Chew E. Parafoveal telangiectasis. In: Ryan SJ, ed. Retina: St. Louis: Elsevier Mosby; 2006:1409-1415.

4. Charbel Issa P, Scholl HPN, Helb HM, Holz FG. Idiopathic macular telangiectasia. In: Holz FG, Spaide RF, eds. Medical Retina. New York: Springer; 2007;183-197.

5. Charbel Issa P, Helb HM, Rohrschneider K, Holz FG, Scholl HPN. Microperimetric assessment of patients with type II macular telangiectasia. Invest Ophthalmol Vis Sci. 2007;48:3788-3795.

6. Cakir M, Kapran Z, Basar D, Utine CA, Eroglu F, Perente I. Optical coherence tomography evaluation of macular edema after intravitreal triamcinolone acetonide in patients with parafoveal telangiectasis. Eur J Ophthalmol. 2006;16:711-717.

7. Alldredge $\mathrm{CD}$, Garretson BR. Intravitreal triamcinolone for the treatment of idiopathic juxtafoveal telangiectasis. Retina. 2003;23: 113-116.

8. Martinez JA. Intravitreal triamcinolone acetonide for bilateral acquired parafoveal telangiectasis. Arch Ophthalmol. 2003;121: $1658-1659$

9. Charbel Issa P, Holz FG, Scholl HPN. Findings in fluorescein angiography and optical coherence tomography after intravitreal bevacizumab in type 2 idiopathic macular telangiectasia. Ophthalmology. 2007;114:1736-1742.

10. Charbel Issa P, Scholl HPN, Holz FG. Intravitreal bevacizumab for the treatment of type 2 idiopathic macular telangiectasis. Retinal Cases Brief Rep. 2007;1:79-81.

11. Jorge R, Costa RA, Calucci D, Scott IU. Intravitreal bevacizumab (Avastin) associated with the regression of subretinal neovascularization in idiopathic juxtafoveolar retinal telangiectasis. Graefes Arch Clin Exp Ophthalmol. 2007;245:1045-1048.

12. Maia Junior OO, Bonanomi MT, Takahashi WY, Nascimento VP, Takahashi BS. Intravitreal bevacizumab for foveal detachment in idiopathic perifoveal telangiectasia. Am J Ophthalmol. 2007;144: 296-299.

13. Mandal S, Venkatesh $\mathrm{P}$, Abbas $\mathrm{Z}$, Vohra R, Garg S. Intravitreal bevacizumab (Avastin) for subretinal neovascularization secondary to type $2 \mathrm{~A}$ idiopathic juxtafoveal telangiectasia. Graefes Arch Clin Exp Ophthalmol. 2007;245:1825-1829.

14. Eandi CM, Ober MD, Freund KB, et al. Anecortave acetate for the treatment of idiopathic perifoveal telangiectasia: a pilot study. Retina. 2006;26:780-785.

15. Gaudric A, Ducos de LG, Cohen SY, Massin P, Haouchine B. Optical coherence tomography in group $2 \mathrm{~A}$ idiopathic juxtafoveolar retinal telangiectasis. Arch Ophthalmol. 2006;124:1410-1419.

16. Paunescu LA, Ko TH, Duker JS, et al. Idiopathic juxtafoveal retinal telangiectasis: new findings by ultrahigh-resolution optical coherence tomography. Ophthalmology. 2006;113:48-57.

17. Surguch V, Gamulescu MA, Gabel VP. Optical coherence tomography findings in idiopathic juxtafoveal retinal telangiectasis. Graefes Arch Clin Exp Ophthalmol. 2007;245:783-788.

18. Cohen SM, Cohen ML, El-Jabali F, Pautler SE. Optical coherence tomography findings in nonproliferative group $2 \mathrm{~A}$ idiopathic juxtafoveal retinal telangiectasis. Retina. 2007;27:59-66.

19. Charbel Issa P, Finger RP, Holz FG, Helb HM, Scholl HPN. A new diagnostic approach in patients with type 2 macular telangiectasia: confocal reflectance-imaging. Acta Ophthalmol Scand. E-pub November 17, 2007. doi:10.1111/j.1600-0420.2007.01041.x.

20. Wustemeyer H, Moessner A, Jahn C, Wolf S. Macular pigment density in healthy subjects quantified with a modified confocal scanning laser ophthalmoscope. Graefes Arch Clin Exp Ophthalmol. 2003;241:647-651.

21. Trieschmann M, Heimes B, Hense HW, Pauleikhoff D. Macular pigment optical density measurement in autofluorescence imaging: comparison of one- and two-wavelength methods. Graefes Arch Clin Exp Ophthalmol. 2006;244:1565-1574. 
22. Snodderly DM, Brown PK, Delori FC, Auran JD. The macular pigment, I: absorbance spectra, localization, and discrimination from other yellow pigments in primate retinas. Invest Opbthalmol Vis Sci. 1984;25:660-673.

23. Behrendt T, Wilson LA. Spectral reflectance photography of the retina. Am J Opbthalmol. 1965;59:1079-1088.

24. Knighton RW, Jacobson SG, Kemp CM. The spectral reflectance of the nerve fiber layer of the macaque retina. Invest Ophthalmol Vis Sci. 1989;30:2392-2402.

25. Radius RL, Anderson DR. The histology of retinal nerve fiber layer bundles and bundle defects. Arch Ophthalmol. 1979;97:948-950.

26. Franze K, Grosche J, Skatchkov SN, et al. Müller cells are living optical fibers in the vertebrate retina. Proc Natl Acad Sci USA. 2007; 104:8287-8292.
27. Daicker B, Schiedt K, Adnet JJ, Bermond P. Canthaxanthin retinopathy: an investigation by light and electron microscopy and physicochemical analysis. Graefes Arch Clin Exp Opbthalmol. 1987;225:189-197.

28. Espaillat A, Aiello LP, Arrigg PG, Villalobos R, Silver PM, Cavicchi RW. Canthaxanthine retinopathy. Arch Opbthalmol. 1999;117: 412- 413 .

29. Boudreault G, Cortin P, Corriveau LA, Rousseau AP, Tardif Y, Malenfant M. La rétinopathie à la canthaxanthine: 1 . Etude clinique de 51 consommateurs. Can J Ophthalmol. 1983;18:325-328.

30. Goralczyk R, Buser S, Bausch J, Bee W, Zuhlke U, Barker FM. Occurrence of birefringent retinal inclusions in cynomolgus monkeys after high doses of canthaxanthin. Invest Opbthalmol Vis Sci. 1997;38:741-752. 